\title{
Erythrodermic paraneoplastic dermatomyositis
}

\author{
Zane J. Brickman MSc, Eric A. Coomes MD, Lynfa Stroud MD
}

— Cite as: CMAJ 2018 December 10;190:E1453. doi: 10.1503/cmaj.180868

A 73-year-old woman presented to the emergency department with erythroderma. A pruritic periorbital rash had spread cephalocaudally over months, eventually involving nearly her entire body surface. Topical corticosteroids, for presumed atopic dermatitis, were ineffective. She subsequently developed proximal weakness and dysphagia, prompting admission to hospital.

Examination showed thickened patches over the extensor surfaces of the patient's interphalangeal joints (Gottron sign) and eyelid erythema with periorbital edema (Figure 1). Her creatinine kinase was elevated (1327 [normal range 30-135] nmol/L). $T_{2}$-weighted magnetic resonance imaging of the quadriceps confirmed active muscular inflammation. Skin biopsy showed perivascular lymphocytic infiltrate, basal vacuolar changes and dermal mucin deposits.

The findings of erythroderma, heliotrope rash and Gottron sign, alongside myositis, suggested dermatomyositis. Antinuclear antibody titers were $>1 / 640$ with anticentromere antibody positivity (other routine serologies, including anti-Jo-1, were negative). There was no interstitial pulmonary fibrosis.

We performed computed tomography of the patient's head, neck, chest and abdomen to assess for malignancy. We identified a gastric mass and on biopsy, confirmed it to be gastric adenocarcinoma. Extended myositis antibody panel showed transcription intermediary factor 1- $\gamma$ (TIF1- $\gamma$ ) antibodies, consistent with cancerassociated dermatomyositis. We began systemic glucocorticoid therapy with prednisone $(1 \mathrm{mg} / \mathrm{kg}$ ), followed by intravenous immunoglobulin and azathioprine. The erythroderma gradually resolved and the patient's functioning recovered. Further considerations for managing the underlying malignancy are underway.

Dermatomyositis is a multisystem inflammatory disorder characterized by proximal weakness with dermatologic manifestations. ${ }^{1}$ Characteristic cutaneous findings include Gottron papules, heliotrope rash and, rarely, erythroderma (diffuse erythema involving most of the body surface)., ${ }^{1,2}$ Supportive investigations include elevated muscle enzymes and classic antibodies, anti-Jo-1 and anti-Mi-2. Extended serologies may predict phenotypes and disease complications. Further evaluation should assess for end-organ consequences, including interstitial lung disease and esophageal dysmotility. Initial management of dermatomyositis consists of glucocorticoid therapy and steroid-sparing agents. ${ }^{1}$

Up to one-third of patients with dermatomyositis are given a diagnosis of cancer, often adenocarcinoma, with peak incidence in the first year after diagnosis. ${ }^{1}$ Antibodies targeted against p155 (TIF1- $\gamma$ antibodies) have a high specificity for cancer-associated dermatomyositis and are associated with severe dermatologic manifestations. ${ }^{3,4}$ Patients should undergo cancer screening.

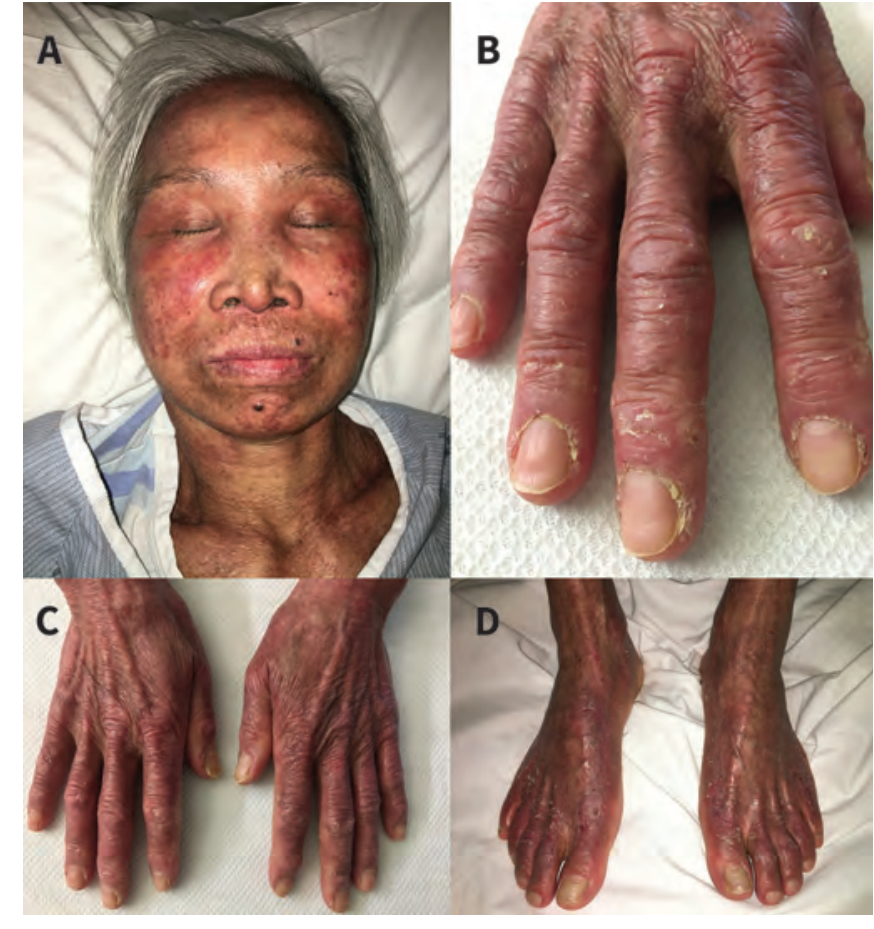

Figure 1: Erythrodermic rash from paraneoplastic dermatomyositis in a 73-year-old woman. A) Eyelid rash with periorbital edema (heliotrope sign). B) Thickened erythematous patches over extensor surfaces of the interphalangeal joints (Gottron sign). C) and D): Erythema.

\section{References}

1. Dalakas MC. Inflammatory muscle diseases. N Engl J Med 2015;372:1734-47.

2. Yuan XY, Guo JY, Dang YP, et al. Erythroderma: a clinical-etiological study of 82 cases. Eur J Dermatol 2010;20:373-7.

3. Selva-O'Callaghan A, Trallero-Araguas E, Grau-Junyent JM, et al. Malignancy and myositis: novel autoantibodies and new insights. Curr Opin Rheumatol 2010;22:627-32.

4. Fiorentino DF, Kuo K, Chung L, et al. Distinctive cutaneous and systemic features associated with antitranscriptional intermediary factor-1 gamma antibodies in adults with dermatomyositis. J Am Acad Dermatol 2015;72:449-55.

\section{Competing interests: None declared.}

This article has been peer reviewed.

The authors have obtained patient consent.

Affiliation: Department of Medicine, University of Toronto, Toronto, Ont.

Correspondence to: Eric Coomes, eric.coomes@mail.utoronto.ca 